Article

\title{
Refractive Index Sensing of Monolayer Molecules Using Both Local and Propagating Surface Plasmons in Mid-Infrared Metagrating
}

\author{
De He, Tieyan Zhang, Lu Liu, Shixing Guo and Zhijun Liu *
}

School of Optoelectronic Science and Engineering, University of Electronic Science and Technology of China, Chengdu 610054, Sichuan, China; vivienhede@126.com (D.H.); 201811050724@std.uestc.edu.cn (T.Z.); liuludian@163.com (L.L.); gsx9303@gmail.com (S.G.)

* Correspondence: liuzhijun@uestc.edu.cn

Received: 10 March 2019; Accepted: 10 April 2019; Published: 12 April 2019

\begin{abstract}
Surface-enhanced infrared absorption spectroscopy (SEIRA) is attractive for molecular sensing due to its high sensitivity and access to molecular fingerprint absorptions. In this paper, we report on refractive index sensing of monolayer molecules in a spectral band outside the molecular fingerprint region. In a metagrating composed of a three-layer metal-insulator-metal structure, both propagating surface plasmon resonances (PSPs) and local surface plasmon resonances (LSPRs) are exited from free-space in a broad band of 3 to $9 \mu \mathrm{m}$, and their sensing properties are characterized. In response to a self-assembled monolayer of octadecanethiol (ODT) molecules, both PSPs and LSPRs exhibit redshifts in wavelength. The shifts of LSPRs are larger than those of PSPs, as originated from their stronger spatial confinement and larger field enhancement. Our proposed mid-infrared molecular sensor is immune to frequency variations of plasmon resonance and more tolerant to sample feature size variation.
\end{abstract}

Keywords: surface plasmon; plasmonic resonance; refractive index sensing; mid-infrared; self-assembled monolayer

\section{Introduction}

Surface plasmons (SPs) are collective oscillations of electrons at the boundaries between materials, which feature strongly confined and intensified fields well suitable for exploring fundamental electromagnetic phenomena [1-5] and for addressing challenges in optical sensing [6-10], and many other applications, such as photovoltaics and lasers [11-13]. Using either propagating or local surface plasmon resonances, which are sensitive to refractive index change of environmental medium, a variety of optical sensors have been demonstrated with sensitivity more than $10^{3} \mathrm{~nm} / \mathrm{RIU}$ in the structures made of metal films [14,15], periodic gratings [16], and nanostructured metal surfaces [17-20].

Although SPs-based sensors thus far have been mostly developed in visible and near-infrared regions, the mid-infrared spectral band has recently attracted considerable interest due to two main reasons [21-24]. One is the presence of the "fingerprint region", which refers to a spectral band having absorption lines of molecular vibration and rotation modes. The unique molecular absorptions can be used to access chemical bond information. The other one is related to the relatively longer wavelength of the mid-infrared. As resonant wavelength in a plasmonic structure is generally scaled with its feature size [25], by moving resonance to a longer wavelength in the mid-infrared, the sample feature size can be made larger, which reduces difficulties in fabrication. Using structures such as metallic nano-rods [26], nano-slits [27], split ring resonators [28], and patterned graphene [29,30] in surface-enhanced infrared absorption spectroscopy (SEIRA), a number of molecular sensors have been 
reported in the mid-infrared, which revealed enhanced fingerprint absorptions of a wealth of molecules. Recently, Mbomson et al. demonstrated asymmetric split H-shape nanoantennas, which showed not only amplified vibrational absorptions of $\mathrm{C}-\mathrm{H}$ bonds but also a large spectral resonance shift with a high sensitivity value of $2335 \mathrm{~nm} /$ RIU [31]. These reported mid-infrared sensors all use local surface plasmon resonances (LSPRs), whose frequencies are generally required to match molecular resonances of target chemical bonds. This frequency matching condition imposes a strict requirement on control over sample feature size in nano-fabrication. One way without this resonance matching requirement is to detect refractive index change caused by molecules in a spectral band outside their fingerprint region, where a significantly wider wavelength range is accessible. This method is, thus, tolerant to frequency variations of plasmon resonance. In this paper, we demonstrate refractive index sensing of molecules outside their fingerprint region using both LSPRs and propagating surface plasmon resonances (PSPs) in a mid-infrared metagrating. Although the mid-infrared plasmon modes are generally considered less spatially-confined as compared to their counterparts in the visible and near-infrared regions, here they are shown to exhibit sensing ability with monolayer sensitivity. In response to a monolayer octadecanethiol (ODT) analyte, both LSPRs and PSPs exhibit clear redshift in wavelength. Our results offer a way of making mid-infrared sensors with reduced restriction on control over sample feature size in fabrication.

\section{Materials and Methods}

Our metagrating structure is illustrated in Figure 1a, which consists of a bottom metal, a ZnSe dielectric layer, and a top metal strip array. LSPRs in such grating are determined by half wavelength resonance condition, which is written as [32]:

$$
\lambda=2 n_{\mathrm{eff}} W / L,(L=1,2,3, \ldots)
$$

where $\lambda$ is the resonance wavelength in free-space, $W$ is the width of gold strip, $L$ is an integer, and $n_{\text {eff }}$ is the effective refractive index. These LSPRs can be regarded as standing waves formed underneath the gold strips. Besides LSPRs, the metagrating also supports PSPs for incident TM polarization, whose excitation is governed by momentum conservation and can be described by [33]:

$$
\frac{2 \pi}{\lambda} n \sin \theta+m \frac{2 \pi}{P}= \pm \frac{2 \pi}{\lambda} \sqrt{\frac{\varepsilon_{m} n^{2}}{\varepsilon_{m}+n^{2}}},(m= \pm 1,2,3, \ldots)
$$

where $\theta$ is the incident angle, $n$ is the refractive index of surrounding environment, which we take as air in our case. $P$ is the period of the grating, $m$ is an integer, and $\varepsilon_{m}$ is the permittivity of gold. In order to excite both LSPRs and PSPs within our interested wavelength range of 3-9 $\mu \mathrm{m}$, the width and period of the metal strips are chosen to be $W=1.3 \mu \mathrm{m}$ and $P=4.5 \mu \mathrm{m}$, respectively. Other designed thickness parameters are given in Figure 1 caption.

To fabricate the structure, we first deposited a $310 \mathrm{~nm}$ thick $\mathrm{Cu}$ film on a Si substrate as the reflector, and then grew a $113 \mathrm{~nm}$ thick $\mathrm{ZnSe}$ film on top of the $\mathrm{Cu}$ using the electron beam evaporation technique. The thickness of ZnSe was measured with ellipsometer SE850 (Sentech, Berlin, Germany). After that, a $105 \mathrm{~nm}$ thick gold strip array was fabricated using stepper photolithography, metal deposition, and lift-off processes. Scanning electron microscope (SEM) image of the fabricated sample is shown in Figure $1 \mathrm{~b}$. The area size of the strip array is $8 \times 8 \mathrm{~mm}$. The geometric parameters of the fabricated sample are listed in Table 1, which were obtained from SEM images and ellipsometry measurements. These sample parameters are in good agreement with the designed parameters in Figure 1a. To add ODT molecules as the analyte, the fabricated sample was immersed in $1 \mathrm{mM} / \mathrm{L}$ ODT (>95\%, Sigma-Aldrich, St. Louis, MO, USA) in ethanol solution for 24 hours at room temperature, and then rinsed thoroughly with ethanol and dried with nitrogen gas. In the self-assembling process, ODT molecules form a monolayer on surfaces of both Au strips and the ZnSe layer [34-36]. A SEM image 
of the sample with monolayer ODT is shown in Figure 1c. Due to some surface imperfections, local aggregation of ODT molecules is evident.

The metagrating sample was characterized with angle-variable Fourier transform infrared (FTIR) reflection spectroscopy [37]. The sample and the detector were mounted on two co-axial rotational stages, which allowed for a continuous scan of the incident angle. The angles were selected from $30^{\circ}$ to $60^{\circ}$ to avoid complexity caused by dispersive fingerprint absorptions of ODT molecules. In such a way, ODT can be regarded as a non-dispersive medium with a constant refractive index. Incident TM polarization with an electrical field perpendicular to the metal strips is used to excite different resonant modes in the measurement. The reflection from a copper mirror was used as the reference background spectrum. To minimize noise arising from environmental disturbance in the optical system, each spectrum was averaged over 64 scans with a resolution of $0.5 \mathrm{~cm}^{-1}$.

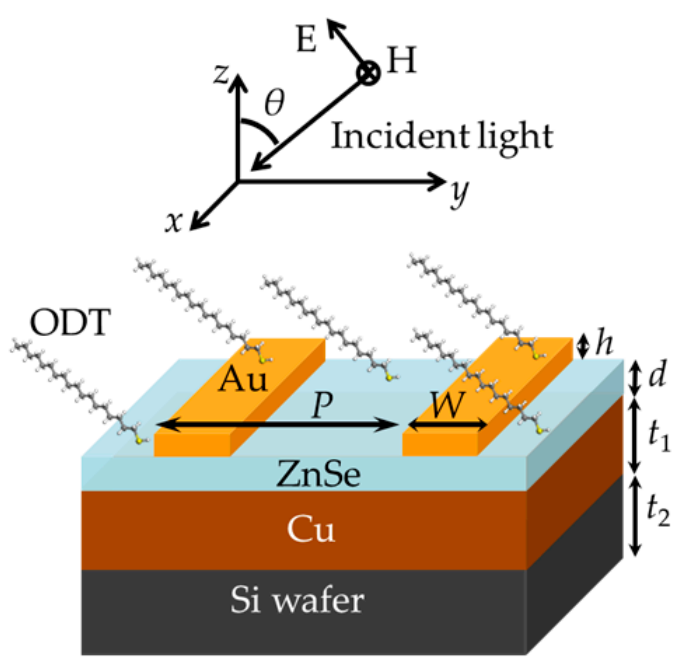

(a)

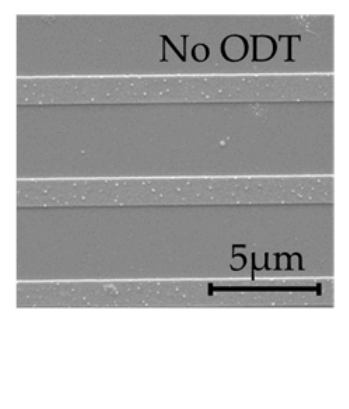

(b)

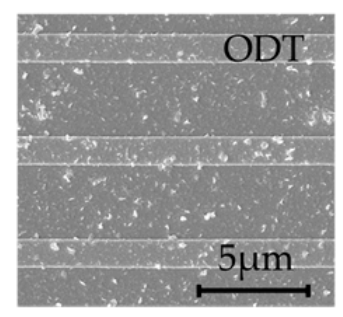

(c)

Figure 1. (a) Schematic of designed metagrating for octadecanethiol (ODT) detection. The relevant dimension parameters are $P=4.5 \mu \mathrm{m}, W=1.3 \mu \mathrm{m}, h=100 \mathrm{~nm}, d=110 \mathrm{~nm}, t_{1}=300 \mathrm{~nm}$, and $t_{2}=500 \mu \mathrm{m}$. The incident light is TM-polarized in $y-z$ plane. Top-view SEM images of the fabricated metagrating before (b) and after (c) ODT adsorption.

Table 1. Geometric parameters of fabricated metagrating sample.

\begin{tabular}{cccccc}
\hline $\boldsymbol{P}(\boldsymbol{\mu m})$ & $W(\mu \mathrm{m})$ & $\boldsymbol{h}(\mathrm{nm})$ & $\boldsymbol{d}(\mathbf{n m})$ & $\boldsymbol{t}_{\mathbf{1}}(\mathrm{nm})$ & $\boldsymbol{t}_{\mathbf{2}}(\boldsymbol{\mu m})$ \\
\hline 4.502 & 1.28 & 105 & 113 & 310 & 520 \\
\hline
\end{tabular}

\section{Results}

Reflection spectra of the sample before ODT adsorption are shown in Figure 2a for different incident angles. At $30^{\circ}$ angle, the reflection spectrum shows four reflection dips, corresponding to four resonant modes. These modes can be understood via their angle-dependent behaviors. The modes at 8.557 and $4.356 \mu \mathrm{m}$ (labeled as L1 and L2) exhibit negligible angle-dependence, which, according to Equation (1), using an effective refractive index of $n_{\text {eff }}=3.29$, are attributed to the LSPRs with mode number $L=1$ and 2, respectively. In addition, the other two modes at 6.714 and $3.385 \mu \mathrm{m}$ (labeled as P1 and P2) show significant redshifts as the angle increases, which are identified as the PSPs with $m=-1$ and -2 , respectively, according to Equation (2). Figure $2 b$ shows a reflectance contour plot of the sample at different angles. The calculated angular dispersions using Equations (1) and (2) are shown in dashed and dotted lines, respectively, which nicely support our analysis of the LSPRs and PSPs.

Figure 3a shows a comparison of reflection spectra of the sample before (black) and after (red) adding the ODT molecules at an incident angle of $30^{\circ}$. From close-up views of the spectra shown in Figure 3b-e, redshifts of the resonances are observed. In particular, the LSPRs, i.e., L1 and L2, 
are shifted by 10 and $9 \mathrm{~nm}$, respectively. Meanwhile, the PSPs, i.e., P1 and P2, are shifted by 2 and $5 \mathrm{~nm}$, respectively. As the angle increases, the wavelength shifts of these four modes are plotted in Figure $4 \mathrm{a}$, and their specific values are listed in Table 2. We can observe an overall trend of redshifts of both LSPRs and PSPs caused by refractive index change of a monolayer ODT. In particular, the P1 and P2 modes exhibit redshifts of $2-5 \mathrm{~nm}$, while the L1 and L2 modes are shifted by $5-16 \mathrm{~nm}$. Therefore, the LSPRs exhibit larger wavelength shifts than the PSPs. In addition, the LSPRs, i.e., L1 and L2, work only in narrow band with wavelength variations of less than $0.193 \mu \mathrm{m}$, while the PSP modes, i.e., P1 and P2, operate in a broad band with a tuned wavelength range of more than $0.782 \mu \mathrm{m}$, as resulted from their different angular dispersions.
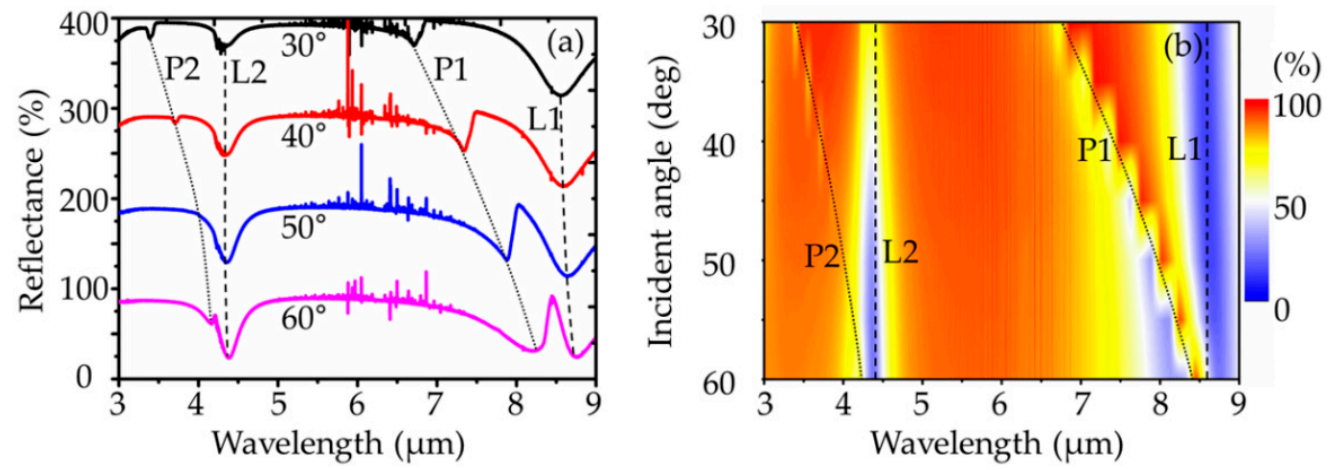

Figure 2. (a) Reflection spectra and (b) reflectance contour plot of the metagrating sample at different incident angles.
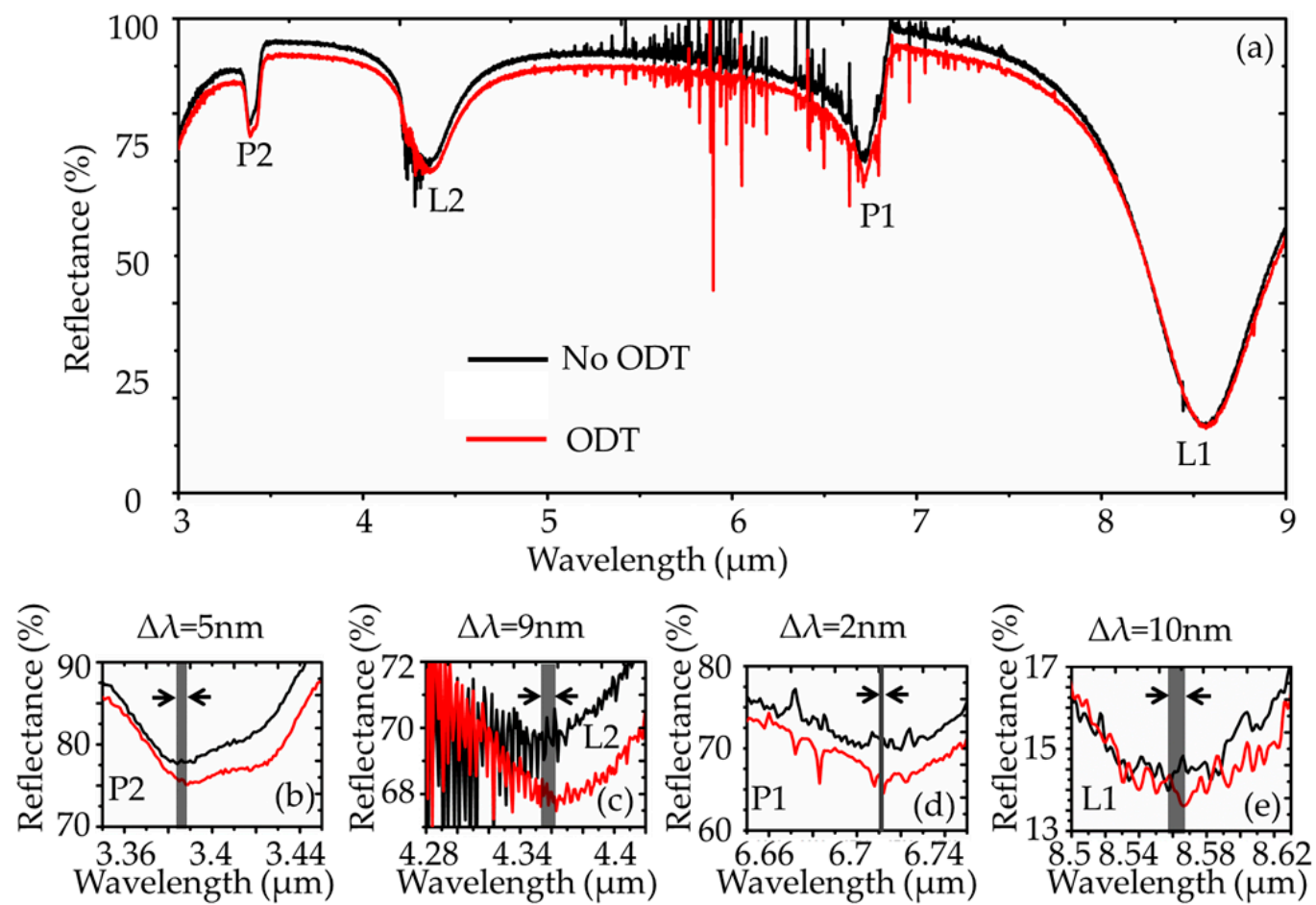

Figure 3. (a) Measured reflection spectra of the metagrating sample with and without octadecanethiol (ODT) at $30^{\circ}$ angle. Close-up view of the spectrum for propagating surface plasmon resonances (PSPs), i.e., (b) P2 with mode number $m=-2$ and (d) P1 with mode number $m=-1$, and local surface plasmon resonances (LSPRs), i.e., (c) L2 with mode number $L=2$ and (e) L1 with mode number $L=1$. 

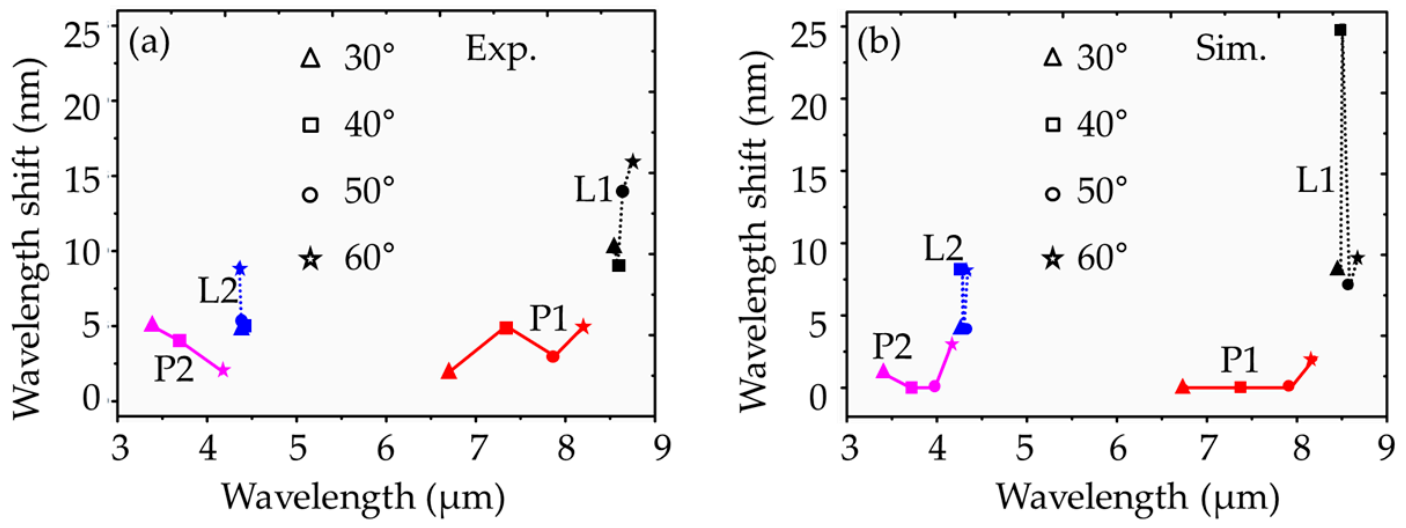

Figure 4. (a) Measured and (b) simulated wavelength shifts of both local surface plasmon resonances (LSPRs) and propagating surface plasmon resonances (PSPs) in the metagrating sample.

Table 2. Measured wavelength shift values of local surface plasmon resonances (LSPRs) and propagating surface plasmon resonances (PSPs) in the metagrating sample.

\begin{tabular}{ccccccccc}
\hline \multirow{2}{*}{$\boldsymbol{\theta}(\mathbf{d e g})$} & \multicolumn{2}{c}{ Mode L1 } & \multicolumn{2}{c}{ Mode P1 } & \multicolumn{2}{c}{ Mode L2 } & \multicolumn{2}{c}{ Mode P2 } \\
\cline { 2 - 8 } & $\boldsymbol{\lambda}(\boldsymbol{\mu \mathbf { m } )}$ & $\boldsymbol{\Delta} \boldsymbol{\lambda}(\mathbf{n m})$ & $\boldsymbol{\lambda}(\boldsymbol{\mu \mathbf { m }})$ & $\boldsymbol{\Delta} \boldsymbol{\lambda}(\mathbf{n m})$ & $\boldsymbol{\lambda}(\boldsymbol{\mu m})$ & $\boldsymbol{\Delta} \boldsymbol{\lambda}(\mathbf{n m})$ & $\boldsymbol{\lambda}(\boldsymbol{\mu m})$ & $\boldsymbol{\Delta} \boldsymbol{( n \mathbf { m } )}$ \\
\hline $30^{\circ}$ & 8.557 & 10 & 6.714 & 2 & 4.356 & 9 & 3.385 & 5 \\
$40^{\circ}$ & 8.586 & 9 & 7.341 & 5 & 4.354 & 9 & 3.698 & 4 \\
$50^{\circ}$ & 8.64 & 14 & 7.881 & 3 & 4.359 & 9 & - & - \\
$60^{\circ}$ & 8.75 & 16 & 8.209 & 5 & 4.383 & 5 & 4.167 & 2 \\
\hline
\end{tabular}

\section{Discussion}

To physically understand the sensing properties of the metagrating sample, we calculated its reflection spectra using a Lumerical FDTD Solver (Lumerical Solutions, Vancouver, BC, Canada). In the calculations, refractive indices of different materials are plotted in Figure 5a, where the $\mathrm{Cu}$ and $\mathrm{Au}$ are described with Drude Model. The plasma frequency and damping frequency of $\mathrm{Cu}$ are taken as $6.38 \times 10^{4} \mathrm{~cm}^{-1}$ and $2.55 \times 10^{2} \mathrm{~cm}^{-1}$, and those of Au are $7.25 \times 10^{4} \mathrm{~cm}^{-1}$ and $2.16 \times 10^{2} \mathrm{~cm}^{-1}$, respectively, as given in a previous study [38]. The refractive index of $\mathrm{ZnSe}$ is taken as 2.42, as reported in another study [39]. For the ODT, its refractive index is considered as a constant of 1.46 [40]. The thickness of ODT is taken as $3 \mathrm{~nm}$, while the geometric dimensions of other layers are given in Figure 1a.

Our simulated wavelength shifts of the four modes in the metagrating are plotted in Figure $4 \mathrm{~b}$. Their detailed wavelength shift values are given in Table 3. These simulated results are in general agreement with the measured results in Figure $4 \mathrm{a}$ and Table 2. There are small quantitative discrepancies in shift values between theory and experiment, which likely originate from uncertainties in optical parameters of materials used in calculations. In addition, the P2 resonance is unresolved in Table 2 due to insufficient signal-to-noise ratio in the measurement. The different sensing behaviors of LSPRs and PSPs can be understood from their field properties, as shown in Figure 5b-e. The LSPRs L1 and L2 are generally more confined in space than the PSPs P1 and P2, and thus the LSPRs have larger field enhancement. The surface field enhancement factor was obtained by calculating the mode field profile first, and then by normalizing the maximum field at the top surface of the metagrating to the incident light. In particular, surface fields of the L1 and L2 modes are enhanced by a factor of 140, while the P1 and P2 modes are enhanced by only 10. Besides this difference in field enhancement, the P1 and P2 modes, on the other hand, exhibit larger effective sensing area with their fields spreading not only on Au strips but also on the ZnSe surface, which is beneficial for accessing more ODT molecules. Overall, the field enhancement is the dominant factor, which explains the better sensitivity of the LSPRs. It is noted that our comparison on sensing properties of LSPRs and PSPs is made for an ultrathin monolayer 
analyte. For bulky targets like thick embedding medium used in earlier reports [14], different results might be expected, since both the field enhancement and the spatial overlap with the analyte molecules likely play significant roles.
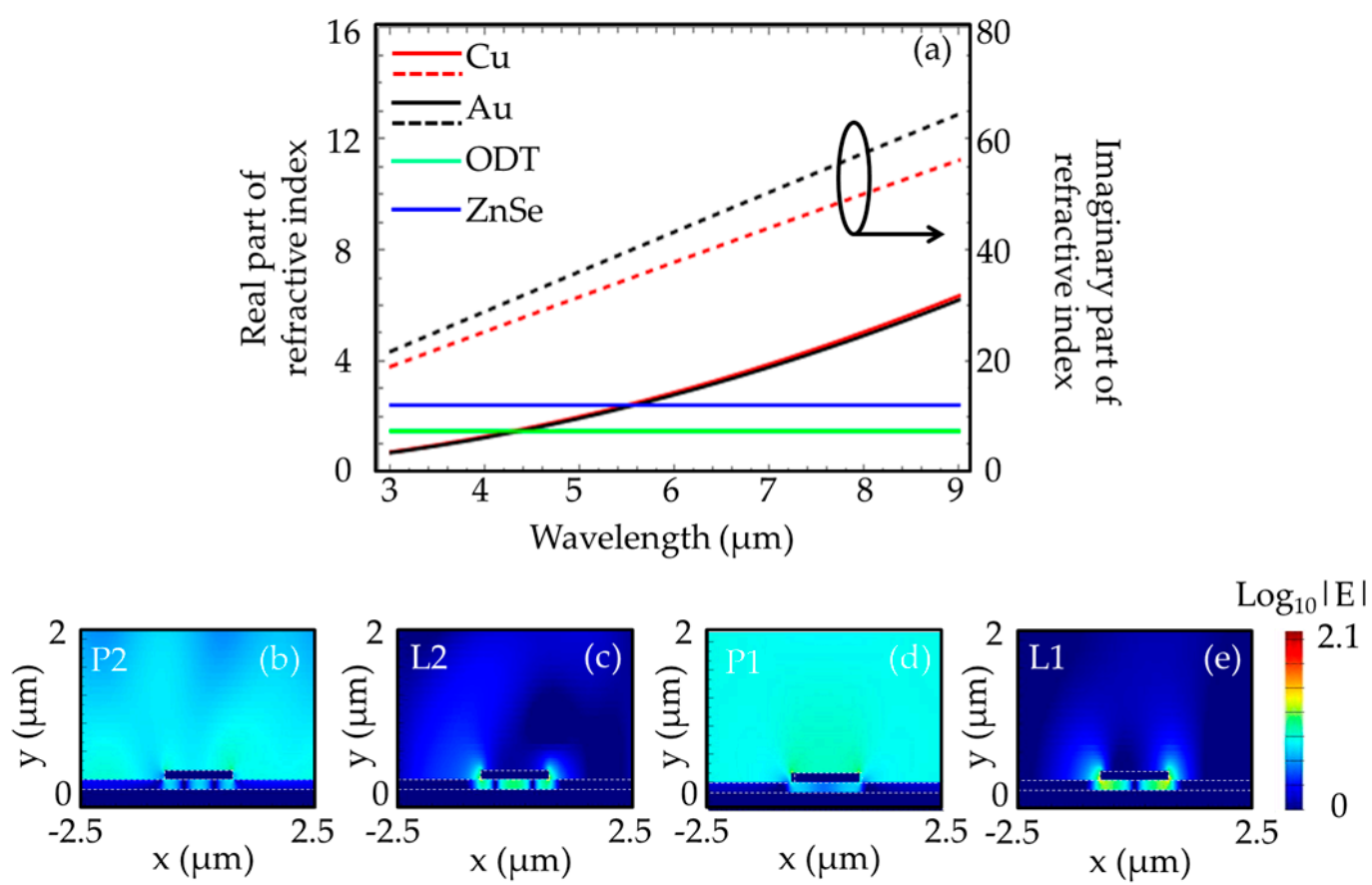

Figure 5. (a) Refractive indices of the relevant materials used in simulation. Spatial field distributions for propagating surface plasmon resonances (PSPs), i.e., (b) P2 with mode number $m=-2$, (d) P1 with mode number $m=-1$, and local surface plasmon resonances (LSPRs), i.e., (c) L2 with mode number $L=2$, (e) L1 with mode number $L=1$.

Table 3. Simulated wavelength shift values of local surface plasmon resonances (LSPRs) and propagating surface plasmon resonances (PSPs) in the metagrating sample.

\begin{tabular}{ccccccccc}
\hline \multirow{2}{*}{$\boldsymbol{\theta}(\mathbf{d e g})$} & \multicolumn{2}{c}{ Mode L1 } & \multicolumn{2}{c}{ Mode P1 } & \multicolumn{2}{c}{ Mode L2 } & \multicolumn{2}{c}{ Mode P2 } \\
\cline { 2 - 9 } & $\boldsymbol{\lambda}(\boldsymbol{\mu \mathrm { m } )}$ & $\boldsymbol{\Delta} \boldsymbol{\lambda}(\mathbf{n m})$ & $\boldsymbol{\lambda}(\boldsymbol{\mu m})$ & $\boldsymbol{\Delta} \boldsymbol{\lambda}(\mathbf{n m})$ & $\boldsymbol{\lambda}(\mu \mathrm{m})$ & $\boldsymbol{\Delta} \boldsymbol{\lambda}(\mathbf{n m})$ & $\boldsymbol{\lambda}(\boldsymbol{\mu m})$ & $\boldsymbol{\Delta} \boldsymbol{\lambda}(\mathbf{n m})$ \\
\hline $30^{\circ}$ & 8.487 & 8 & 6.759 & 0 & 4.285 & 4 & 3.402 & 1 \\
$40^{\circ}$ & 8.505 & 25 & 7.392 & 0 & 4.284 & 8 & 3.708 & 0 \\
$50^{\circ}$ & 8.584 & 7 & 7.931 & 0 & 4.302 & 4 & 3.976 & 0 \\
$60^{\circ}$ & 8.678 & 9 & 8.192 & 2 & 4.34 & 8 & 4.163 & 3 \\
\hline
\end{tabular}

Finally, it is interesting to quantify the sensing performance of both LSPRs and PSPs in terms of Figure of Merit (FOM). Since the analyte in our structure is an ultrathin monolayer instead of an entire embedding medium, it is challenging to obtain the actual change of refractive index, which depends on not only the optical constant of the ODT molecules but also the spatial coupling of the monolayer film into the volume of the plasmon field. The regularly defined FOM $=\Delta \lambda /(\Delta n \Gamma)$, as well as sensitivity $(\Delta \lambda /(\Delta n))$ [41], where $\Delta \lambda$ is the wavelength shift, $\Delta n$ is the change of refractive index, and $\Gamma$ is the width of the resonance, cannot be determined in experiment for our case of monolayer analyte. In fact, Becker et al. proposed an alternative definition of FOM for thin-film target [41]:

$$
\mathrm{FOM} *_{\text {layer }}=|d I / I| / d l
$$

where $\mathrm{dI} / \mathrm{I}$ is the maximum relative intensity change at a fixed wavelength induced by spectral shift of the resonance, and $d l$ is the thickness of the analyte thin-film. This defined $\mathrm{FOM}^{*}$ layer captures the effective sensing volumes of various nanostructures, and we used it in our sample characterization. 
The experimentally determined FOM ${ }^{*}$ layer values of both LSPRs and PSPs in our sample are plotted in Figure 6. It is seen that the LSPRs L1 and L2 exhibit a maximum FOM ${ }_{\text {layer }}$ of 0.18 , while the PSPs P1 and P2 have a smaller FOM* ${ }_{\text {layer }}$ of less than 0.1 .

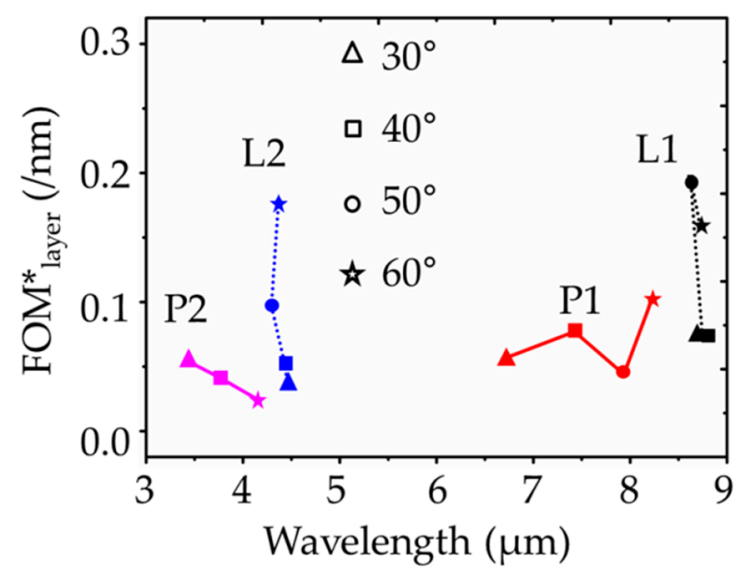

Figure 6. Experimental figure of merit $\mathrm{FOM}^{*}$ layer of different modes in the metagrating sample.

\section{Conclusions}

We demonstrated refractive index sensing of monolayer molecules outside their fingerprint region using both LSPRs and PSPs in a mid-infrared metagrating. Within a broad band of 3 to $9 \mu \mathrm{m}$, PSPs with mode numbers $L=1,2$, and LSPRs with mode numbers $m=-1,-2$ were excited and their sensing properties were examined. In response to monolayer ODT molecules, the PSPs exhibited wavelength redshifts of 2-5 nm, while the LSPRs showed wavelength redshifts in the range of 5-16 nm. The larger redshifts of LSPRs result from their larger surface field enhancement, as compared with the PSPs. In addition, the LSPR modes worked at essentially fixed wavelengths, while the PSPs modes operated in a broad band with a wavelength tuning range of more than $0.782 \mu \mathrm{m}$, as originated from their different angular dispersions. Our proposed metagrating sensor relies on refractive index change caused by analyte molecules in a spectral band without fingerprint absorptions. This approach is less sensitive to frequency variations of plasmon resonance, and thus is advantageous in terms of fabrication tolerance.

Author Contributions: Conceptualization, D.H. and Z.L.; methodology, D.H. and S.G.; formal analysis, D.H., T.Z. and L.L.; writing—original draft preparation, D.H.; writing—review and editing, Z.L.; supervision, Z.L.; funding acquisition, Z.L.

Funding: This research was funded by National Natural Science Foundation of China (NSFC) under grant numbers 61875030, 61575036, and 61421002.

Acknowledgments: The authors acknowledge the fabrication facility of State Key Laboratory of Electronic Thin Films and Integrated Devices at University of Electronic Science and Technology of China.

Conflicts of Interest: The authors declare no conflict of interest.

\section{References}

1. Wood, R.W. On a remarkable case of uneven distribution of light in a diffraction grating spectrum. Phil. Mag. 1902, 4, 396-402. [CrossRef]

2. Rayleigh, L. On the dynamical theory of gratings. Proc. R. Soc. London Ser. A 1907, 79, 399-416. [CrossRef]

3. Otto, A. Excitation of nonradiative surface plasma waves in silver by the method of frustrated total reflection. Z. Phys. 1968, 216, 398-410. [CrossRef]

4. Kretschmann, E.; Raether, H. Radiative decay of non-radiative surface plasmons excited by light. Z. Naturforsch. A 1968, 23, 2135-2136. [CrossRef]

5. Barnes, W.L.; Dereux, A.; Ebbesen, T.W. Surface plasmon subwavelength optics. Nature 2003, 424, 824-830. [CrossRef] [PubMed] 
6. Homola, J.; Piliarik, M. Surface plasmon resonance (SPR) sensors. Springer Ser. Chem. Sens. Biosens. 2006, 4, 45-67.

7. Mayer, K.M.; Hafner, J.H. Localized surface plasmon resonance sensors. Chem. Rev. 2011, 111, 3828-3857. [CrossRef] [PubMed]

8. Ignatyeva, D.O.; Knyazev, G.A.; Kapralov, P.O.; Dietler, G.; Sekatskii, S.K.; Belotelov, V.I. Magneto-optical plasmonic heterostructure with ultranarrow resonance for sensing applications. Sci. Rep. 2016, 6, 28077. [CrossRef]

9. Rizal, C.; Pisana, S.; Hrvoic, I. Improved magneto-optic surface plasmon resonance biosensors. Photonics 2018, 5, 15. [CrossRef]

10. Rizal, C. Microstructure, surface plasmon, magneto-optic surface plasmon, and sensitivity properties of magneto-plasmonic Co/Au multilayers. IEEE T. Magn. 2018, 54, 5300309. [CrossRef]

11. Tvingstedt, K.; Persson, N.K.; Inganäs, O. Surface plasmon increase absorption in polymer photovoltaic cells. Appl. Phys. Lett. 2007, 91, 113514. [CrossRef]

12. Atwater, H.A.; Polman, A. Plasmonics for improved photovoltaic devices. Nat. Mater. 2010, 9, $205-213$. [CrossRef] [PubMed]

13. Berini, P.; Leon, I.D. Surface plasmon-polariton amplifiers and lasers. Nat. Photonics 2012, 6, 16-24. [CrossRef]

14. Haes, A.J.; Duyne, R.P.V. A unified view of propagating and localized surface plasmon resonance biosensors. Anal. Bioanal. Chem. 2004, 379, 920-930. [CrossRef]

15. Jung, L.S.; Campbell, C.T.; Chinowsky, T.M. Quantitative interpretation of the response of surface plasmon resonance sensors to adsorbed films. Langmuir 1998, 14, 5636-5648. [CrossRef]

16. Alleyne, C.J.; Kirk, A.G.; McPhedran, R.C.; Nicorovici, N.P.; Maystre, D. Enhanced SPR sensitivity using periodic metallic structures. Opt. Express 2007, 15, 8163-8169. [CrossRef] [PubMed]

17. Shen, Y.; Zhou, J.; Liu, T.; Tao, Y.; Jiang, R.; Liu, M.; Xiao, G.; Zhu, J.; Zhou, Z.; Wang, X. Plasmonic gold mushroom arrays with refractive index sensing figures of merit approaching the theoretical limit. Nat. Commun. 2013, 4, 2381. [CrossRef] [PubMed]

18. Petryayeva, E.; Krull, U.J. Localized surface plasmon resonance: Nanostructures, bioassays and biosensing-A review. Anal. Chim. Acta. 2011, 706, 8-24. [CrossRef]

19. Willets, K.A.; Duyne, R.P.V. Localized surface plasmon resonance spectroscopy and sensing. Annu. Rev. Phys. Chem. 2007, 58, 267-297. [CrossRef]

20. Christ, A.; Zentgraf, T.; Tikhodeev, S.G.; Gippius, N.A.; Kuhl, J.; Giessen, H. Controlling the interaction between localized and delocalized surface plasmon modes: Experiment and numerical calculations. Phys. Rev. B 2006, 74, 155435. [CrossRef]

21. Cheng, F.; Yang, X.; Gao, J. Enhancing intensity and refractive index sensing capability with infrared plasmonic perfect absorbers. Opt. Lett. 2014, 39, 3185-3188. [CrossRef]

22. Mayerhöfer, T.G.; Popp, J. Periodic array-based substrates for surface enhanced infrared spectroscopy. Nanophotonics 2018, 7, 39-79. [CrossRef]

23. Vogt, J.; Huck, C.; Neubrech, F.; Toma, A.; Gerbert, D.; Pucci, A. Impact of the plasmonic near- and far-field resonance-energy shift on the enhancement of infrared vibrational signals. Phys. Chem. Chem. Phys. 2015, 17, 21169-21175. [CrossRef]

24. Huck, C.; Vogt, J.; Sendner, M.; Hengstler, D.; Neubrech, F.; Pucci, A. Plasmonic enhancement of infrared vibrational signals: nanoslits versus nanorods. ACS Photonics 2015, 2, 1489-1497. [CrossRef]

25. Novotny, L. Effective wavelength scaling for optical antennas. Phys. Rev. Lett. 2007, 98, 266802. [CrossRef]

26. Neubrech, F.; Pucci, A.; Cornelius, T.W.; Karim, S.; Garcia-Etxarri, A.; Aizpurua, J. Resonant plasmonic and vibrational coupling in a tailored nano antenna for infrared detection. Phys. Rev. Lett. 2008, 101, 157403. [CrossRef] [PubMed]

27. Huck, C.; Toma, A.; Neubrech, F.; Chirumamilla, M.; Vogt, J.; Angelis, D.F.; Pucci, A. Gold nano antennas on a pedestal for plasmonic enhancement in the infrared. ACS Photonics 2015, 2, 497-505. [CrossRef]

28. Lahiri, B.; Khokhar, A.Z.; De La Rue, R.M.; McMeekin, S.G.; Johnson, N.P. Asymmetric split ring resonators for optical sensing of organic materials. Opt. Express 2009, 17, 1107-1115. [CrossRef] [PubMed]

29. Rodrigo, D.; Limaj, O.; Janner, D.; Etezadi, D.; de-Abajo, F.J.G.; Pruneri, V.; Altug, H. Mid-infrared plasmonic biosensing with graphene. Sicence 2015, 349, 165-168. [CrossRef] 
30. Zhu, Y.; Li, Z.; Hao, Z.; DiMarco, C.; Maturavongsadit, P.; Hao, Y.; Lu, M.; Stein, A.; Wang, Q.; Hone, J. Optical conductivity-based ultrasensitive mid-infrared biosensing on a hybrid metasurface. Light-Sci. Appl. 2018, 7, 67.

31. Mbomson, I.G.; Tabor, S.; Lahiri, B.; Sharp, G.; McMeekin, S.G.; De La Rue, R.M.; Johnson, N.P. Asymmetric split H-shape nanoantennas for molecular sensing. Biomed. Opt. Express 2017, 8, 395-406. [CrossRef]

32. Todorov, Y.; Tosetto, L.; Teissier, J.; Andrews, A.M.; Klang, P.; Colombelli, R.; Sagnes, I.; Strasser, G.; Sirtori, C. Optical properties of metal-dielectric-metal micro cavities in the THz frequency range. Opt. Expres. 2010, 18, 13886-13907. [CrossRef] [PubMed]

33. Homola, J.; Koudela, I.; Yee, S.S. Surface plasmon resonance sensors based on diffraction gratings and prism couplers: sensitivity comparison. Sensors Actuat. B 1999, 54, 16-24. [CrossRef]

34. Enders, D.; Pucci, A. Surface enhanced infrared absorption of octadecanethiol on wet-chemically prepared Au nanoparticle films. Appl. Phys. Lett. 2006, 88, 184104. [CrossRef]

35. Noble-Luginbuhl, A.R.; Nuzzo, R.G. Assembly and characterization of SAMs formed by the adsorption of alkanethiols on zinc selenide substrates. Langmuir 2001, 17, 3937-3944. [CrossRef]

36. He, D.; Guo, S.; Liu, L.; Zhang, T.; Jiang, Y.; Xu, J.; Liu, Z. Amplified molecular detection sensitivity in passive dielectric cavity. Opt. Express 2018, 26, 32026-32036. [CrossRef]

37. Stuart, B. Infrared Spectroscopy: Fundamentals and Applications; John Wiley \& Sons, Ltd.: Sussex, UK, 2004.

38. Ordal, M.A.; Long, L.L.; Bell, R.J.; Bell, S.E.; Bell, R.R.; Alexander, R.W., Jr.; Ward, C.A. Optical properties of the metals $\mathrm{Al}, \mathrm{Co}, \mathrm{Cu}, \mathrm{Au}, \mathrm{Fe}, \mathrm{Pb}, \mathrm{Ni}, \mathrm{Pd}, \mathrm{Pt}, \mathrm{Ag}, \mathrm{Ti}$, and $\mathrm{W}$ in the infrared and far infrared. Appl. Optics 1983, 22, 1099-1119. [CrossRef]

39. Ahmed, S.; Khawaja, E.E. Refractive index of thin films of ZnSe in the IR. Thin Solid Films. 1984, 112, L1-L4. [CrossRef]

40. Lide, D.R. Handbook of Chemistry and Physics; CRC Press: Boca Raton, FL, USA, 2005.

41. Becker, J.; Trügler, A.; Jakab, A.; Hohenester, U.; Sönnichsen, C. The optimal aspect ratio of gold nanorods for plasmonic bio-sensing. Plasmonics 2010, 5, 161-167. [CrossRef]

(C) 2019 by the authors. Licensee MDPI, Basel, Switzerland. This article is an open access article distributed under the terms and conditions of the Creative Commons Attribution (CC BY) license (http://creativecommons.org/licenses/by/4.0/). 